\title{
Chemical Composition of the Atmospheres of Red Giants with High Space Velocities
}

\author{
Yu. V. Pakhomov ${ }^{1}$ \\ ${ }^{1}$ Institute of Astronomy, Russian Academy of Sciences, \\ Pyatnitskaya ul. 48, Moscow, 109017 Russia, pakhomov@inasan.ru
}

\begin{abstract}
The results of a comparative analysis of the elemental abundances in the atmospheres of 14 red giants with high Galactic space velocities are presented. For almost all of the chemical elements considered, the their abundance trends with metallicity correspond to those constructed for thickdisk dwarfs. In the case of sodium, the main factor affecting the $[\mathrm{Na} / \mathrm{Fe}]$ abundance in the stellar atmosphere for red giants is the surface gravity that characterizes the degree of development of the convective envelope. The difference between the $[\mathrm{Na} / \mathrm{Fe}]$ abundances in the atmospheres of thin-and thick-disk red giants has been confirmed.

Keywords: stellar spectroscopy, stellar atmospheres, red giants, stellar evolution, kinematics, Galactic chemical evolution.

PACS: 97.10.Cv, 97.10.Ex, 97.10.Ri, 97.10.Tk, 97.20.Li, 98.35.Bd, 98.35.Df, 98.35.Pr
\end{abstract}

\section{INTRODUCTION}

Our Galaxy is currently believed to be a set of subsystems that differ by their sizes, locations in the Galaxy, characteristic stellar velocities, and stellar populations. The best-known subsystems are the nucleus, the bulge, the thick and thin disks, and the halo. Each subsystem can contain dynamical stellar groups or streams (see, e.g., [23], 24]). At a Galactocentric distance of about $8.5 \mathrm{kpc}$, where the Solar system is located, there are stars of at least three Galactic subsystems: the thick and thin disks and the halo. In addition, stars with significant orbital eccentricities that can belong to the bulge exist in the inner Galaxy. Such stars are characterized by an enhanced metallicity, and super-metalrich stars are more often observed among the bulge objects [40]. In contrast, the halo stars exhibit a low metallicity and a large scatter of orbital inclinations and eccentricities. Thus, the structure and chemical evolution of the Galaxy can be investigated by studying the motion of stars in the solar neighborhood, their physical characteristics, and the abundances of chemical elements in their atmospheres.

The chemical composition of the stellar atmospheres changes as the star on the temperature. luminosity diagram passes from the main sequence to the red giant branch in the course of its evolution. The hydrogen burning reactions cease in the core and begin in the shell around the core. At this stage of its life, the structure of the star changes significantly: the star rapidly expands, and an extensive convective shell that provides the first deep mixing of stellar matter is formed. The nuclear burning products are brought into the stellar atmosphere and become observable. The changes in the CNO abundances are well known: carbon and oxygen are reduced approximately by $0.1-0.3$ dex, while nitrogen is enhanced by 0.1-0.4 dex. In addition to these elements, the changes can also affect other elements.

In this paper, we analyze the chemical composition of red giants with Galactic velocities that exceed those typical of thin-disk stars in the solar neighborhood and the main causes of the changes in elemental abundances.
TABLE I: The list of studied stars with the membership probabilities $(p)$ in some Galactic subsystems

\begin{tabular}{|c|c|c|c|c|c|c|c|c|}
\hline $\mathrm{N}$ & HD & $\begin{array}{l}\alpha_{2000} \\
\mathrm{~h}: \mathrm{m}: \mathrm{s} \\
\end{array}$ & $\begin{array}{c}\delta_{2000} \\
\circ: \prime: / \prime\end{array}$ & $m_{V}$ & SpType & $\begin{array}{l}\text { thin } \\
\text { disc } \\
\end{array}$ & $\begin{array}{c}p, \% \\
\text { Hercules } \\
\text { stream }\end{array}$ & $\begin{array}{l}\text { thick } \\
\text { disc }\end{array}$ \\
\hline 1 & 2901 & $00: 32: 47.6$ & $+54: 07: 11$ & 6.91 & K2III & - & - & 99 \\
\hline 2 & 10550 & 01:42:43.5 & $-03: 41: 24$ & 4.98 & K3II-III & 27 & 58 & 13 \\
\hline 3 & 92095 & 10:39:05.7 & $+53: 40: 05$ & 5.55 & K3III & 16 & - & 83 \\
\hline 4 & 94600 & $10: 55: 44.4$ & $+33: 30: 25$ & 5.02 & K1III & 91 & - & 8 \\
\hline 5 & 94669 & $10: 56: 14.5$ & $+42: 00: 29$ & 6.03 & K2III & 30 & 41 & 27 \\
\hline 6 & 94860 & $10: 59: 56.8$ & $+77: 46: 12$ & 6.18 & G9III & 15 & 4 & 80 \\
\hline 7 & 99902 & $11: 29: 41.9$ & $+15: 24: 47$ & 5.74 & K4III & 87 & - & 11 \\
\hline 8 & 100696 & $11: 36: 02.7$ & $+69: 19: 22$ & 5.19 & K0III & 52 & - & 47 \\
\hline 9 & 104985 & $12: 05: 15.1$ & $+76: 54: 20$ & 5.78 & G9III & 38 & - & 61 \\
\hline 10 & 127243 & $14: 28: 37.8$ & $+49: 50: 41$ & 5.58 & G3IV & - & - & 99 \\
\hline 11 & 141353 & $15: 48: 13.3$ & $+13: 47: 21$ & 5.98 & K2III & 1 & 1 & 97 \\
\hline 12 & 141472 & $15: 46: 34.7$ & $+55: 28: 29$ & 5.94 & K3III & - & - & 99 \\
\hline 13 & 152879 & $16: 55: 22.2$ & $+18: 25: 59$ & 5.35 & K4III & 4 & - & 95 \\
\hline 14 & 197752 & $20: 44: 52.5$ & $+25: 16: 14$ & 4.92 & K2III & 41 & - & 58 \\
\hline
\end{tabular}

These changes reflect not only the chemical evolution of the Galactic subsystem to which they belong but also the stellar evolution processes.

\section{OBSERVATIONS}

We selected the stars for our observations based on their Galactic velocities calculated from reduced Hipparcos parallaxes [42] and CORAVEL radial velocities 36]. We chose the red giants for which at least one of the Galactic velocity vector components (UVW) exceeded its typical values for the thin disk $(34.5,22.5$, 18.0) $\mathrm{km} \mathrm{s}^{-1}$ [42]. The effective temperatures $T_{\text {eff }}$ and surface gravities lg $g$ for the program stars were preestimated from photometric data and reduced Hipparcos parallaxes [42]. Subsequently, the stars with effective temperatures $T_{\text {eff }}$ from 4000 to $5200 \mathrm{~K}$ and surface grav- 
TABLE II: Atmospheric parameters of the investigated stars, their physical parameters, and interstellar extinctions

\begin{tabular}{|c|c|c|c|c|c|c|c|c|}
\hline $\mathrm{N}$ & $\mathrm{HD}$ & $\begin{array}{c}T_{\text {eff }} \\
\mathrm{K}\end{array}$ & $\lg g$ & $\begin{array}{c}V_{t} \\
{k m s^{-1}}^{-1}\end{array}$ & $\begin{array}{c}\mathrm{Fe} / \mathrm{H}] \\
M_{\odot}\end{array}$ & $\begin{array}{c}\text { Mass } \\
\text { lg, years }\end{array}$ & $\begin{array}{c}A_{V} \\
\mathrm{~m}\end{array}$ \\
\hline 1 & 2901 & 4350 & 2.15 & 1.25 & -0.46 & $0.7 \pm 0.3$ & $9.4 \pm 0.5$ & 0.1 \\
2 & 10550 & 4290 & 1.25 & 1.50 & 0.05 & $7 \pm 1$ & $7.7 \pm 0.4$ & 0.1 \\
3 & 92095 & 4430 & 2.15 & 1.38 & -0.02 & $1.9 \pm 0.3$ & $9.0 \pm 0.3$ & $<0.1$ \\
4 & 94600 & 4660 & 2.50 & 1.20 & -0.05 & $1.7 \pm 0.3$ & $9.0 \pm 0.3$ & 0 \\
5 & 94669 & 4620 & 2.65 & 1.25 & 0.00 & $1.5 \pm 0.4$ & $8.9 \pm 0.2$ & 0 \\
6 & 94860 & 4970 & 2.75 & 1.20 & -0.03 & $2.8 \pm 0.3$ & $8.7 \pm 0.2$ & $<0.1$ \\
7 & 99902 & 4380 & 2.25 & 1.30 & 0.22 & $1.9 \pm 0.5$ & $9.0 \pm 0.3$ & $<0.1$ \\
8 & 100696 & 4920 & 2.70 & 1.26 & -0.21 & $2.4 \pm 0.3$ & $8.6 \pm 0.3$ & 0 \\
9 & 104985 & 4830 & 2.80 & 1.28 & -0.10 & $1.9 \pm 0.3$ & $9.0 \pm 0.3$ & 0 \\
10 & 127243 & 5100 & 1.75 & 1.28 & -0.44 & $1.6 \pm 0.3$ & $9.1 \pm 0.3$ & 0 \\
11 & 141353 & 4280 & 1.95 & 1.37 & -0.09 & $2.0 \pm 0.4$ & $9.0 \pm 0.4$ & $<0.1$ \\
12 & 141472 & 4180 & 1.50 & 1.40 & -0.27 & $1.1 \pm 0.3$ & $9.0 \pm 0.5$ & $<0.1$ \\
13 & 152879 & 4170 & 1.80 & 1.39 & 0.02 & $1.4 \pm 0.5$ & $9.5 \pm 0.5$ & $<0.1$ \\
14 & 197752 & 4570 & 2.25 & 1.33 & 0.03 & $2.6 \pm 0.5$ & $8.8 \pm 0.5$ & $<0.1$ \\
\hline
\end{tabular}

ities $\lg g$ from 0.5 to 3.0 were chosen from the list. The list of program stars is presented in Table [.

The spectroscopic observations of the chosen stars were performed in 2007-2008 with a two-arc echelle spectrograph attached to a 2.16-m telescope at the Xinglong station of the National Astronomical Observatories of China (NAOC). The spectrograph operated in the redband mode. The detector was a $1024 \times 1024$ CCD array on which 40 spectral orders in the range from 5565 to $9194 \AA$ were recorded. The spectrograph resolution was $R=40000$; the signal-to-noise ratio in the spectra was $S / N>150$.

The echelle package of the MIDAS software system was used for the spectroscopic data reduction, the search for and extraction of the spectral orders, the wavelength calibration using the spectrum of a thorium-argon lamp, and the spectrum normalization. The equivalent widths of the selected spectral lines were measured in the EW code that I wrote.

\section{DETERMINING THE PARAMETERS OF THE STELLAR ATMOSPHERES}

Table II gives the parameters of the stellar atmospheres (effective temperature $T_{e f f}$, surface gravity $\lg g$, microturbulence $V_{t}$, and metallicity $[\mathrm{Fe} / \mathrm{H}]$ ), the stellar masses and ages on a logarithmic scale, and the interstellar extinctions $A_{V}$. We determined the masses and ages based on the evolutionary tracks from [28] by taking into account the stellar metallicity. The interstellar extinctions were estimated from the color excesses $\mathrm{E}(B-V)$, $\mathrm{E}(V-J), \mathrm{E}(V-H), \mathrm{E}(V-K), \mathrm{E}(b-y)$ the normal colors were calculated from the calibrations by [8], which are based on Kurucz.s model stellar atmospheres [15].

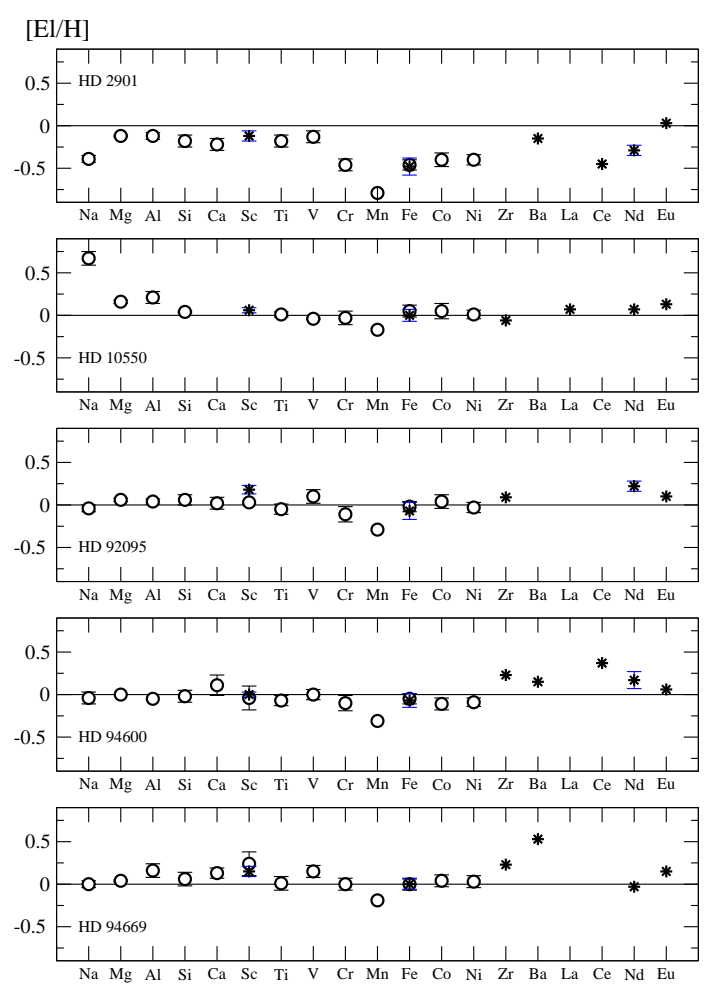

FIG. 1: Relative elemental abundances in the atmospheres of the program stars. The open circles and asterisks denote the abundances determined from spectral lines of neutral and ionized atoms, respectively.

We determined the parameters of the stellar atmospheres using a technique that is also based on Kurucz.s model atmospheres [15] and analysis of the relative abundances of iron-peak elements. The technique is described in detail in [9] and allows the parameters of the stellar atmospheres for G-K giants to be determined with an accuracy of about $70-100 \mathrm{~K}$ for $T_{e f f}, 0.10-0.15$ for $\lg g$, and $0.10-0.15 \mathrm{~km} \mathrm{~s}^{-1}$ for $V_{t}$. In this paper, when analyzing the relative abundances of iron-peak elements when determining the parameters of the stellar atmospheres, we disregarded titanium, because it is well known that the $[\mathrm{Ti} / \mathrm{Fe}]$ abundance can be enhanced at low metallicities and for thick-disk stars [7]. Using the derived parameters, we computed the corresponding model stellar atmospheres with the ATLAS9 code [33]. Based on the measured equivalent widths of the selected unblended spectral lines, we estimated the elemental abundances with the WIDTH9 code. These are presented in Table III and Figure 1, where the open circles and asterisks denote the abundances determined from the spectral lines of neutral and ionized atoms, respectively. The list of selected lines with their characteristics and equivalent widths is available in electronic form. The cobalt abundance was determined by taking into account the hyperfine splitting effect, which can be strong in the case of cool giants [10]. The abundance errors given inTable III and marked by the bars in Figure 1 were determined as 
TABLE III: Elemental abundances in the atmospheres of the program stars

\begin{tabular}{|c|c|c|c|c|c|c|c|c|c|c|c|c|c|c|}
\hline & \multicolumn{2}{|r|}{ HD 2901} & \multicolumn{2}{|r|}{ HD 10550} & \multicolumn{2}{|r|}{ HD 92095} & \multicolumn{2}{|r|}{ HD 94600} & \multicolumn{2}{|r|}{ HD 94669} & \multicolumn{2}{|r|}{ HD 94860} & \multicolumn{2}{|c|}{ HD 99902} \\
\hline & $\mathrm{N}$ & {$[\mathrm{El} / \mathrm{H}]$} & $\mathrm{N}$ & {$[\mathrm{El} / \mathrm{H}]$} & $\mathrm{N}$ & {$[\mathrm{El} / \mathrm{H}]$} & $\mathrm{N}$ & {$[\mathrm{El} / \mathrm{H}]$} & $\mathrm{N}$ & {$[\mathrm{El} / \mathrm{H}]$} & $\mathrm{N}$ & {$[\mathrm{El} / \mathrm{H}]$} & $\mathrm{N}$ & {$[\mathrm{El} / \mathrm{H}]$} \\
\hline $\mathrm{NaI}$ & 2 & $.39 \pm 0.04$ & 2 & $0.67 \pm 0.08$ & 2 & $0.04 \pm 0.04$ & 2 & $.04 \pm 0.07$ & 2 & $0.00 \pm 0.04$ & 2 & $0.27 \pm 0.02$ & 2 & $0.40 \pm 0.04$ \\
\hline MgI & 2 & 01 & 2 & .02 & 2 & 02 & 2 & 0.00 & 2 & .01 & 2 & 0.02 & 2 & 0.08 \\
\hline $\mathrm{AlI}$ & 2 & $.12 \pm 0.04$ & 2 & 0.21 & 2 & .03 & 2 & 0.00 & 2 & 0.08 & 2 & $.26 \pm 0.01$ & 2 & $=0.05$ \\
\hline $\mathrm{SiI}$ & 8 & .07 & 2 & 01 & 7 & 0.06 & 10 & 0.07 & 9 & 0.08 & 8 & $=0.03$ & 8 & $29 \pm 0.08$ \\
\hline $\mathrm{CaI}$ & 3 & $.22 \pm 0.07$ & - & & 3 & $0.02 \pm 0.07$ & 4 & $0.11 \pm 0.12$ & 3 & $.13 \pm 0.06$ & 4 & $13 \pm 0.02$ & 3 & $0.29 \pm 0.06$ \\
\hline $\mathrm{ScI}$ & - & - & - & - & 1 & & 2 & 0.14 & 2 & 0.14 & 1 & & - & - \\
\hline ScII & 5 & $.12 \pm 0.06$ & 2 & $0.06 \pm 0.03$ & 4 & $0.18 \pm 0.05$ & 3 & .03 & 5 & .06 & 3 & .03 & 4 & $0.28 \pm 0.10$ \\
\hline TiI & 20 & $.18 \pm 0.07$ & 5 & $0.01 \pm 0.03$ & 20 & -0.05 & 25 & 0.06 & 24 & 0.08 & 24 & .05 & 14 & 0.06 \\
\hline VI & 7 & .07 & 2 & -0.04 & 7 & 0.10 & 20 & .06 & 13 & .07 & 25 & .07 & 3 & 0.07 \\
\hline CrI & 6 & .07 & 4 & -0.03 & 7 & .09 & 11 & 0.09 & 10 & 0.07 & 9 & \pm 0.05 & 8 & $=0.09$ \\
\hline $\mathrm{MnI}$ & 1 & -0.79 & 1 & -0 & 1 & -0 & 1 & & & & - & - & 1 & 3 \\
\hline $\mathrm{FeI}$ & 66 & $-0.46 \pm 0.06$ & 16 & $0.05 \pm 0.07$ & 58 & $-0.02 \pm 0.06$ & 73 & $-0.05 \pm 0.06$ & 63 & $0.00 \pm 0.07$ & 66 & $-0.03 \pm 0.06$ & 55 & 0.07 \\
\hline FeII & 6 & $0.48 \pm 0.10$ & 4 & $0.00 \pm$ & 6 & $-0.07 \pm 0.10$ & 5 & -0.0 & 5 & 0.00 & 5 & -0.0 & 2 & .03 \\
\hline $\mathrm{CoI}$ & 4 & $.40=$ & 4 & 0.05 & 4 & 0.04 & 5 & .07 & 0 & 0.04 & 5 & 12 & 8 & .09 \\
\hline $\mathrm{NiI}$ & 21 & $.40 \pm$ & 6 & $0.01=$ & 13 & -0.03 & 18 & 05 & 14 & 0.03 & 18 & .07 & 14 & .06 \\
\hline YII & . & - & - & - & - & - & - & - & - & - & 1 & -0.32 & 2 & 0.03 \\
\hline 11 & - & - & 1 & -0.06 & 1 & 0 & 1 & 0.20 & 1 & 0.20 & - & - & 1 & \\
\hline 01 & 2 & $.42 \pm 0.02$ & - & - & 1 & & - & - & - & - & 1 & & 1 & 0.06 \\
\hline I & & -0.15 & - & - & 1 & & 1 & & 1 & & 1 & & - & - \\
\hline I & & - & 1 & & - & - & - & - & - & - & - & - & 1 & 0 \\
\hline II & 1 & -0.4 & - & . & 1 & & 1 & & 1 & & 1 & & 1 & \\
\hline dII & 4 & 0.29 & 1 & & 3 & 06 & 2 & & 1 & & - & - & 2 & 0.02 \\
\hline \multirow[t]{3}{*}{ EuII } & 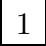 & & 1 & & 1 & & 1 & & 1 & & 1 & & 1 & \\
\hline & \multicolumn{2}{|c|}{ HD 100696} & \multicolumn{2}{|c|}{ HD 104985} & \multicolumn{2}{|c|}{ HD 127243} & \multicolumn{2}{|c|}{ HD 141353} & \multicolumn{2}{|r|}{ HD 141472} & \multicolumn{2}{|c|}{ HD 152879} & \multicolumn{2}{|c|}{ HD 197752} \\
\hline & $\mathrm{N}$ & {$[\mathrm{El} / \mathrm{H}]$} & $\mathrm{N}$ & & $\mathrm{N}$ & & $\mathrm{N}$ & & $\mathrm{N}$ & & $\mathrm{N}$ & & $\mathrm{N}$ & \\
\hline & 2 & & 2 & & 3 & & 2 & & 2 & & 2 & & 2 & \\
\hline $\mathrm{MgI}$ & 2 & 2 & 2 & & 2 & 03 & 2 & & 2 & & 2 & & 2 & .03 \\
\hline $\mathrm{AlI}$ & 2 & & 2 & & 2 & & 2 & & 2 & & 1 & & 2 & .08 \\
\hline $\mathrm{SiI}$ & 10 & 08 & 13 & 05 & 10 & 07 & 8 & & 8 & .06 & 7 & & 6 & 0.04 \\
\hline $\mathrm{CaI}$ & 4 & & 3 & 0.06 & 4 & .05 & 3 & & 4 & .05 & 2 & & 3 & 0.05 \\
\hline $\mathrm{ScI}$ & 2 & .11 & 1 & & 1 & & - & - & 1 & & - & - & 1 & \\
\hline ScII & 4 & $0.14 \pm 0.07$ & 5 & $0.25=$ & 3 & $-0.37 \pm 0.07$ & 5 & $2 \pm 0.09$ & 5 & 0.07 & 3 & & 6 & 0.04 \\
\hline TiI & 24 & $.19 \pm 0.06$ & 20 & 0.03 & 15 & $-0.27 \pm$ & 17 & $-0.0^{\prime}$ & 13 & .05 & 9 & .08 & 20 & 0.06 \\
\hline VI & 26 & $-0.20 \pm 0.08$ & 18 & 0.07 & 8 & -0.44 & 3 & 0.05 & 3 & -0.1 & 1 & & 6 & 0.05 \\
\hline $\mathrm{CrI}$ & 8 & $0.21 \pm 0.08$ & 7 & -0.1 & 1 & & 11 & $=0.11$ & 4 & 0.04 & 6 & & 11 & $=0.04$ \\
\hline MnI & 1 & & 1 & & 1 & & 1 & & 1 & & - & - & 1 & \\
\hline $\mathrm{FeI}$ & 79 & $-0.21 \pm 0.06$ & 69 & 0.06 & 53 & $-0.51 \pm 0.08$ & 57 & $-0.09 \pm 0.07$ & 51 & $-0.33 \pm 0.08$ & 38 & & 62 & 0.05 \\
\hline FeII & 6 & $-0.20 \pm 0.08$ & 5 & $-0.12 \pm 0.08$ & 6 & $-0.57 \pm 0.07$ & 4 & & 3 & & 2 & & 4 & 0.04 \\
\hline $\mathrm{CoI}$ & 4 & $-0.27 \pm 0.05$ & 5 & $-0.02 \pm 0.07$ & 2 & $-0.39 \pm 0.00$ & 6 & & 6 & -0.2 & 4 & & 5 & 0.08 \\
\hline $\mathrm{NiI}$ & 25 & $-0.22 \pm 0.07$ & 20 & $-0.06 \pm 0.05$ & 13 & $-0.50 \pm 0.07$ & 11 & & 9 & $-0.30 \pm 0.05$ & 6 & $=0.07$ & 15 & $=0.06$ \\
\hline YII & 1 & & - & - & - & - & 1 & & 2 & $-0.21 \pm 0.02$ & 1 & 1.20 & 1 & \\
\hline & 1 & & - & - & - & - & 1 & & 1 & -0.06 & - & - & 1 & \\
\hline BaII & 1 & 0.09 & 1 & 0.14 & 1 & -0.36 & 1 & 0.14 & - & - & - & - & 1 & 0.29 \\
\hline & - & - & - & - & - & - & - & - & 1 & 10 & - & - & 1 & 0.47 \\
\hline & 2 & $10 \pm 0$ & 1 & & - & - & 2 & 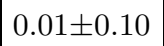 & 3 & $0.03 \pm 0.09$ & 2 & 10 & 2 & $0.10 \pm 0.06$ \\
\hline EuII & 1 & -0.08 & 1 & 0.20 & 1 & -0.31 & 1 & 0.09 & 1 & -0.03 & 1 & 0.09 & 1 & 0.12 \\
\hline
\end{tabular}


$[\mathrm{El} / \mathrm{H}]$
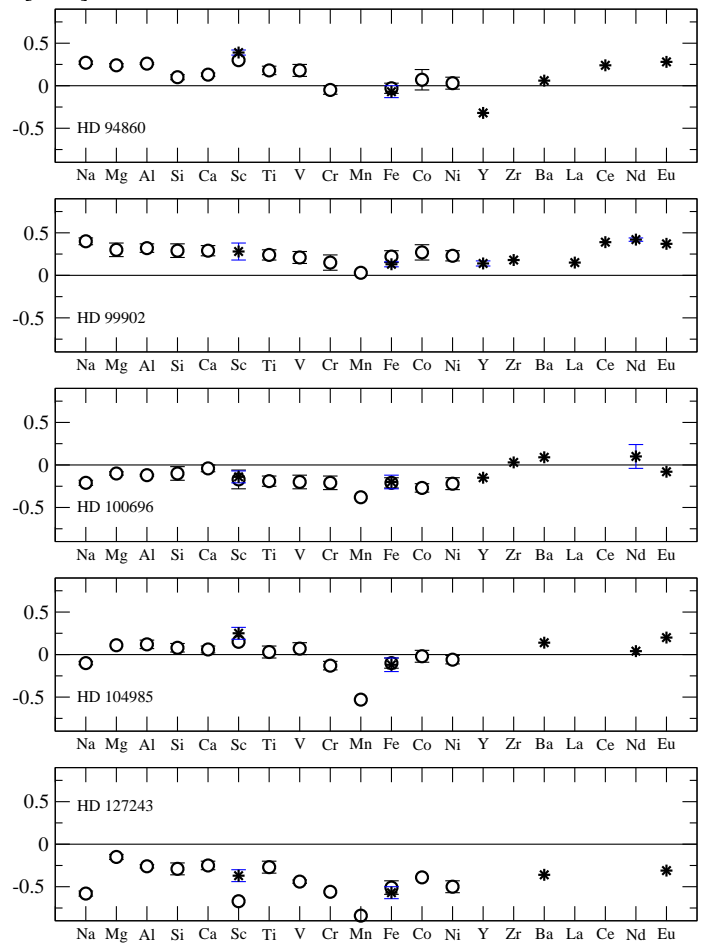
[El/H]

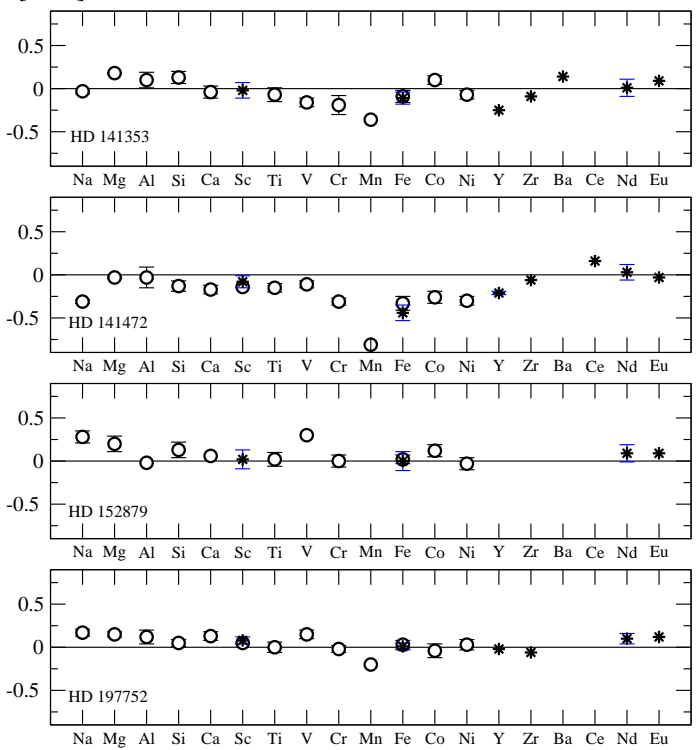

FIG. 1: Relative elemental abundances in the atmospheres of the program stars. The open circles and asterisks denote the abundances determined from spectral lines of neutral and ionized atoms, respectively. (continue)

the dispersion of the individual abundances calculated from individual spectral lines. The possible abundance errors associated with the determination of stellar atmosphere parameters are listed in Table IV for the stars HD $100673\left(T_{e f f}=4920 \mathrm{~K} \mathrm{lg} g=2.70 V_{t}=1.26 \mathrm{~km} \mathrm{~s}^{-1}\right)$ and HD $152879\left(T_{e f f}=4170 \mathrm{~K} 80 V_{t}=1.39 \mathrm{~km} \mathrm{~s}^{-1}\right)$ as an
TABLE IV: Changes of the elemental abundances in the atmospheres of the stars HD $100673\left(T_{\text {eff }}=4920 \mathrm{~K}\right.$ $\left.\lg g=2.70 V_{t}=1.26 \mathrm{~km} \mathrm{~s}^{-1}\right)$ HD $152879\left(T_{e f f}=4170 \mathrm{~K}\right.$ $\lg g=1.80 V_{t}=1.39 \mathrm{~km} \mathrm{~s}^{-1}$ ) when changing the model parameters by $\Delta T_{\text {eff }}=+100 \mathrm{~K}, \Delta \lg g=+0.10$, $\Delta V_{t}=+0.10 \mathrm{~km} \mathrm{~s}^{-1}$ and the total change in abundance $\Delta$

\begin{tabular}{|c|c|c|c|c|c|c|c|c|c|c|}
\hline \multirow[t]{2}{*}{ Elem } & \multirow[t]{2}{*}{$\mathrm{N}$} & \multicolumn{4}{|c|}{$\Delta[E l / H]_{H D 100673}$} & \multirow[t]{2}{*}{$\mathrm{N}$} & \multicolumn{4}{|c|}{$\Delta[E l / H]_{H D 152879}$} \\
\hline & & $\Delta T_{e f f}$ & $\Delta \lg g$ & $\Delta V_{t}$ & $\Delta$ & & $\Delta T_{e f f}$ & $\Delta \lg g$ & $\Delta V_{t}$ & $\Delta$ \\
\hline NA1 & 2 & 0.07 & 0.00 & -0.02 & 0.07 & 2 & 0.07 & -0.01 & -0.05 & 0.09 \\
\hline MG1 & 2 & 0.05 & 0.00 & -0.01 & 0.05 & 2 & -0.03 & 0.01 & -0.03 & 0.0 \\
\hline AL1 & 2 & 0.05 & 0.00 & -0.01 & 0.05 & 1 & 0.06 & 0.00 & -0.03 & $0.0^{\prime}$ \\
\hline SI1 & 10 & -0.02 & 0.01 & -0.02 & 0.03 & 7 & -0.10 & 0.03 & -0.02 & 0.1 \\
\hline CA1 & 4 & 0.10 & 0.00 & -0.04 & 0.11 & 2 & 0.11 & 0.00 & -0.04 & 0.12 \\
\hline $\mathrm{SC} 1$ & 2 & 0.14 & 0.00 & -0.01 & 0.14 & & & & & \\
\hline $\mathrm{SC} 2$ & 4 & -0.01 & 0.05 & -0.02 & 0.05 & 3 & -0.03 & 0.04 & -0.03 & 0.06 \\
\hline TI1 & 24 & 0.12 & -0.01 & -0.02 & 0.12 & 9 & 0.11 & 0.00 & -0.04 & $0.1:$ \\
\hline V1 & 26 & 0.15 & 0.00 & -0.02 & 0.15 & 1 & 0.13 & 0.01 & -0.04 & 0.1 \\
\hline CR1 & 8 & 0.09 & 0.00 & -0.01 & 0.09 & 6 & 0.09 & 0.01 & -0.03 & 0.10 \\
\hline MN1 & 1 & 0.07 & 0.00 & 0.00 & 0.07 & & & & & \\
\hline FE1 & 79 & 0.07 & 0.01 & -0.02 & 0.07 & 38 & -0.02 & 0.03 & -0.03 & 0.05 \\
\hline FE2 & 6 & -0.08 & 0.05 & -0.04 & 0.10 & 2 & -0.22 & 0.07 & -0.04 & 0.2 \\
\hline $\mathrm{CO} 1$ & 4 & 0.09 & 0.02 & 0.00 & 0.09 & 4 & 0.00 & 0.03 & -0.02 & 0.04 \\
\hline NI1 & 25 & 0.05 & 0.01 & -0.03 & 0.06 & 6 & -0.04 & 0.03 & -0.04 & 0.06 \\
\hline Y2 & 1 & -0.01 & 0.05 & 0.00 & 0.05 & 1 & -0.03 & 0.03 & -0.03 & 0.05 \\
\hline ZR2 & 1 & -0.01 & 0.05 & 0.00 & 0.05 & & & & & \\
\hline BA2 & 1 & 0.02 & 0.03 & -0.11 & 0.12 & & & & & \\
\hline ND2 & 2 & 0.01 & 0.05 & 0.00 & 0.05 & 2 & 0.01 & 0.03 & -0.02 & 0.04 \\
\hline EU2 & & -0.01 & 0.05 & -0.01 & 0.05 & 1 & -0.01 & 0.04 & -0.02 & 10.0 \\
\hline
\end{tabular}

example. Table IV gives the number of lines used $N$ and the changes in the abundance of each element when changing individual model parameters $\left(\Delta T_{\text {eff }}=+100 \mathrm{~K}\right.$, $\left.\Delta \lg g=+0.10, \Delta V_{t}=+0.10 \mathrm{~km} \mathrm{~s}^{-1}\right)$ and the total change in abundance.

\section{STELLAR KINEMATICS}

The last columns in Table 1 give the membership probabilities of the program stars in Galactic subsystems whose kinematic characteristics were taken from [25]. We calculated the probabilities based on the formulas from [37]. Table 1 includes the thick and thin disks and the Hercules stellar stream, because it turned out that some of the stars could also belong to the latter. In our previous paper [38] devoted to the Hercules stellar stream, we showed that the stream is inhomogeneous in its composition and could contain both thin-and thick-disk stars. The membership probability in the halo and other subsystems and moving stellar groups are not given in Table I. because it turned out to be less than $1 \%$ for all program stars.

Table $\mathrm{V}$ presents the kinematic characteristics of 
TABLE V: Kinematic parameters of the investigated stars

\begin{tabular}{|c|c|c|c|c|c|c|c|c|}
\hline $\mathrm{N}$ & $\mathrm{HD}$ & $\begin{array}{c}U \\
\mathrm{ks}^{-1}\end{array}$ & $\begin{array}{c}V \\
\mathrm{~km} \mathrm{~s}^{-1}\end{array}$ & $\begin{array}{c}W \\
\mathrm{~km} \mathrm{~s}^{-1}\end{array}$ & $\begin{array}{c}R_{\min } \\
\mathrm{kpc}\end{array}$ & $\begin{array}{c}R_{\max } \\
\mathrm{kpc}\end{array}$ & $\begin{array}{c}Z_{\max } \\
\mathrm{kpc}\end{array}$ & $e$ \\
\hline 1 & 2901 & $24.4 \pm 13.0$ & $-144.5 \pm 9.1$ & $-36.9 \pm 8.9$ & $2.17 \pm 0.16$ & $8.67 \pm 0.03$ & $0.42 \pm 0.10$ & $0.60 \pm 0.02$ \\
2 & 10550 & $-57.5 \pm 3.4$ & $-42.1 \pm 2.7$ & $3.0 \pm 2.2$ & $6.11 \pm 0.07$ & $9.97 \pm 0.07$ & $0.36 \pm 0.00$ & $0.24 \pm 0.01$ \\
3 & 92095 & $67.6 \pm 2.3$ & $-54.2 \pm 3.2$ & $22.1 \pm 0.6$ & $5.62 \pm 0.08$ & $9.42 \pm 0.04$ & $0.46 \pm 0.01$ & $0.25 \pm 0.01$ \\
4 & 94600 & $20.5 \pm 0.6$ & $-18.3 \pm 0.4$ & $-36.9 \pm 0.4$ & $8.17 \pm 0.01$ & $8.74 \pm 0.01$ & $0.41 \pm 0.00$ & $0.03 \pm 0.00$ \\
5 & 94669 & $-41.7 \pm 0.7$ & $-43.3 \pm 1.6$ & $-38.2 \pm 0.5$ & $6.19 \pm 0.04$ & $9.39 \pm 0.02$ & $0.45 \pm 0.00$ & $0.21 \pm 0.00$ \\
6 & 94860 & $20.4 \pm 2.5$ & $-59.9 \pm 1.5$ & $-43.9 \pm 0.7$ & $5.73 \pm 0.04$ & $8.62 \pm 0.01$ & $0.55 \pm 0.01$ & $0.20 \pm 0.01$ \\
7 & 99902 & $-3.3 \pm 0.3$ & $-23.8 \pm 2.0$ & $-39.3 \pm 0.8$ & $7.77 \pm 0.06$ & $8.75 \pm 0.02$ & $0.47 \pm 0.01$ & $0.06 \pm 0.00$ \\
8 & 100696 & $-40.7 \pm 0.6$ & $-20.4 \pm 0.3$ & $35.8 \pm 0.6$ & $7.24 \pm 0.01$ & $10.01 \pm 0.02$ & $0.76 \pm 0.01$ & $0.16 \pm 0.00$ \\
9 & 104985 & $-76.2 \pm 1.6$ & $-8.5 \pm 0.3$ & $30.5 \pm 1.1$ & $6.91 \pm 0.02$ & $12.15 \pm 0.04$ & $0.74 \pm 0.02$ & $0.28 \pm 0.00$ \\
10 & 127243 & $78.6 \pm 2.0$ & $-111.0 \pm 2.7$ & $53.0 \pm 1.5$ & $3.30 \pm 0.05$ & $9.15 \pm 0.02$ & $1.21 \pm 0.03$ & $0.47 \pm 0.01$ \\
11 & 141353 & $-8.9 \pm 3.3$ & $-69.9 \pm 4.1$ & $-56.3 \pm 1.3$ & $5.17 \pm 0.11$ & $8.47 \pm 0.01$ & $0.81 \pm 0.02$ & $0.24 \pm 0.01$ \\
12 & 141472 & $155.0 \pm 11.7$ & $-71.2 \pm 5.1$ & $63.7 \pm 5.1$ & $4.10 \pm 0.10$ & $12.74 \pm 0.37$ & $1.96 \pm 0.13$ & $0.51 \pm 0.02$ \\
13 & 152879 & $10.1 \pm 0.8$ & $-28.5 \pm 1.6$ & $58.3 \pm 2.4$ & $7.87 \pm 0.06$ & $8.41 \pm 0.00$ & $1.22 \pm 0.04$ & $0.03 \pm 0.00$ \\
14 & 197752 & $-78.1 \pm 3.2$ & $-6.6 \pm 1.6$ & $-44.7 \pm 1.8$ & $6.88 \pm 0.04$ & $12.23 \pm 0.10$ & $0.67 \pm 0.02$ & $0.28 \pm 0.01$ \\
\hline
\end{tabular}

the program stars. These include the Galactic velocity vector $(U, V, W)$ relative to the Sun and the Galactic orbital elements: the perigalactic distance $R_{\text {min }}$, the apogalactic distance $R_{\max }$, the maximum orbital distance from the Galactic plane $Z_{\max }$, the eccentricity $e$, and the inclination $i$. The distance to the Galactic center was assumed to be $8.5 \mathrm{kpc}$, while the necessary correction of the velocities for the solar motion, $(+10.2,+14.9,+7.8) \mathrm{km} \mathrm{s}^{-1}$, was taken from [25]. We calculated the orbital elements through numerical integration of the stellar motion by Everhart.s 15th-order method using a three-component model Galactic potential [1]. The integration accuracy was controlled by the conservation of the necessary integrals of motion. For example, in ten orbital revolutions, the typical relative error was $\Delta h / h<10^{-13}$ in angular momentum and $\Delta E / E<10^{-8}$ in total energy. The errors in the space velocities $(\sigma U, \sigma V, \sigma W)$ were calculated from the errors in the stellar proper motions, radial velocities, parallaxes and the errors in the solar velocity components relative to the local standard of rest. We calculated the errors in the Galactic orbital elements based on the model Galactic gravitational potential using the probable errors in the stellar space velocities.

We see from Table \ that the membership probability in the thin disk at least for two stars (HD 94600 and HD 99902) exceeds considerably that for the thick disk and these probabilities are almost equal for one star (HD 100696). However, these stars were also included in our program, because the maximum orbital distance from the Galactic plane $Z_{\max }$ for them exceeds the characteristic scale height for thin-disk objects, 90-325 pc (27], [41], 20]).

\section{DISCUSSION}

Figure 2 presents the $[\mathrm{Na} / \mathrm{Fe}],[\mathrm{Mg} / \mathrm{Fe}],[\mathrm{Al} / \mathrm{Fe}],[\mathrm{Si} / \mathrm{Fe}]$, $[\mathrm{Ca} / \mathrm{Fe}]$, and $[\mathrm{Ti} / \mathrm{Fe}]$ abundance trends with metallicity in the atmospheres of the red giants investigated here (the large circles with ordinal numbers from Table 【i) in comparison with other stars. The small filled circles indicate the 74 thin-disk red giants from [3 6, 10, 14, 38 -40] that we investigated by a unified technique. The filled and open triangles indicate the thin-disk (29 stars) and thick-disk (22 stars) red giants from [2], which is devoted to the investigation of the Galactic chemical evolution in the solar neighborhood. The solid and dashed lines indicate the thin-and thick-disk dwarfs from [7] averaged with a metallicity interval of 0.2 dex, while the shaded regions denote a dispersion of $1 \sigma$.

The six chemical elements in Fig. 2 are among the indicators of the Galactic chemical evolution, with four of them being represented by the -elements $\mathrm{Mg}, \mathrm{Si}, \mathrm{Ca}$, and Ti. We see from Fig. 2 that the behavior of all elements, except sodium, in the atmospheres of the red giants is similar to that for the dwarfs.

Figure 3 presents the s-element $[\mathrm{Y} / \mathrm{Fe}]$ and $[\mathrm{Ba} / \mathrm{Fe}]$ and r-element $[\mathrm{Eu} / \mathrm{Fe}]$ abundance trends with metallicity. The designations in the figure are similar to those in Fig. 2. This figure does not present all stars. For example, the Y II lines in some cases are too weak for their equivalent widths to be properly measured, while the $\mathrm{Ba}$ II lines, on the contrary, often have an equivalent width larger than 100-150 $\mathrm{mA}$. In this case, the line wings and the significantly growing influence of non-LTE processes play a great role. All of this makes it impossible to determine the barium abundance in this technique with an accuracy characteristic of other elements (about $0.1 \mathrm{dex}$ ). 
$[\mathrm{El} / \mathrm{Fe}]$

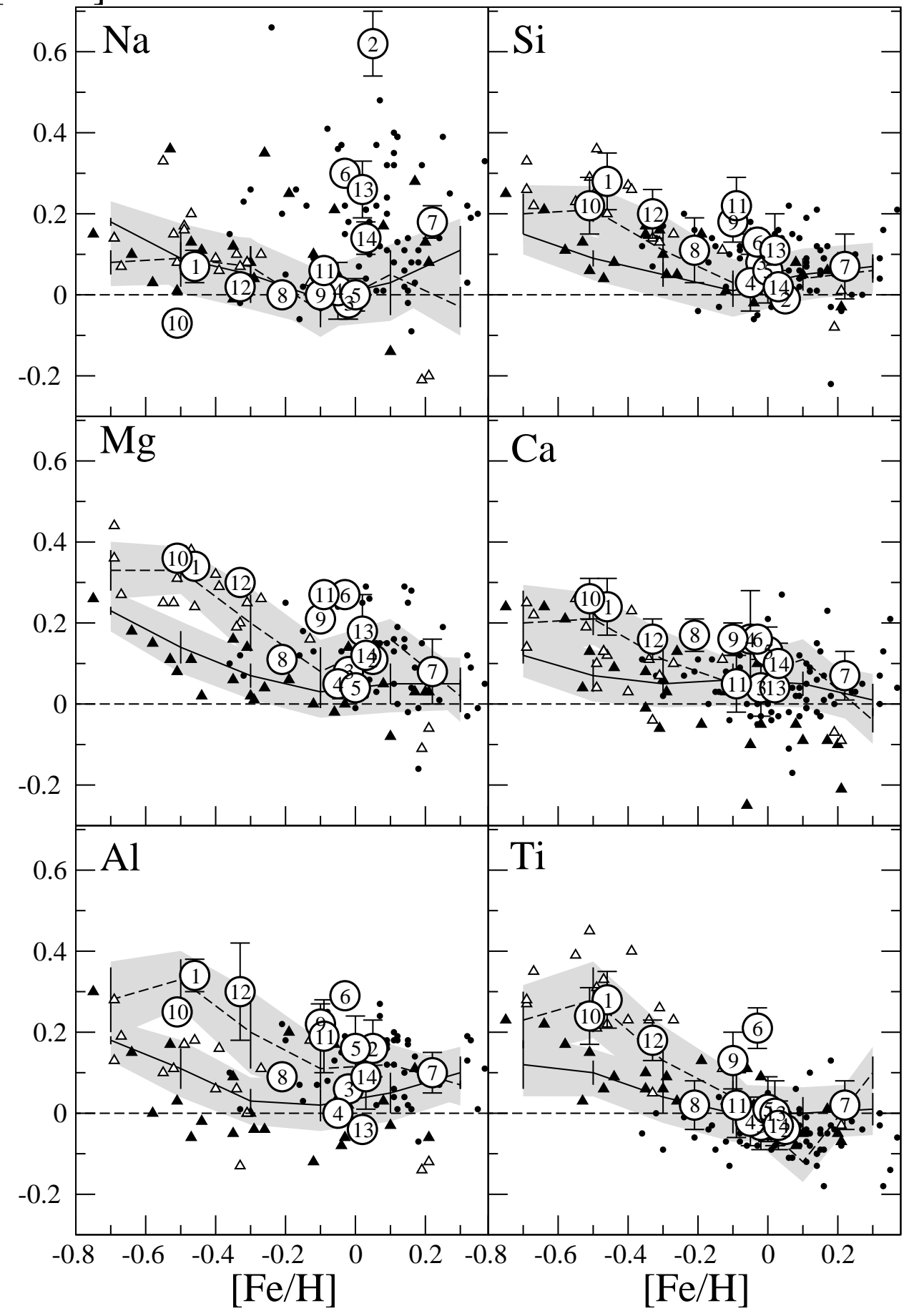

FIG. 2: $[\mathrm{Na}, \mathrm{Mg}, \mathrm{Al}, \mathrm{Si}, \mathrm{Ca}, \mathrm{Ti} / \mathrm{Fe}]$ abundance trends with metallicity in the atmospheres of the red giants investigated here (the large circles with the star numbers from Table I) in comparison with the thin-disk red giants that we studied previously (filled small circles), the thin-and thick-disk red giants from [2] (filled and open triangles), and the thin- and thick-disk dwarfs from [7] (the solid and dashed lines in the shaded regions).

\section{Sodium}

The sodium abundance was determined from the $\mathrm{Na}$ I 6154 and $6160 \AA$ lines without any correction for non-
LTE processes. According to [32], 35], 34], this doublet is formed deeper than other sodium lines, and the nonLTE processes do not introduce significant corrections to the abundance determination. We see from Fig. 2 


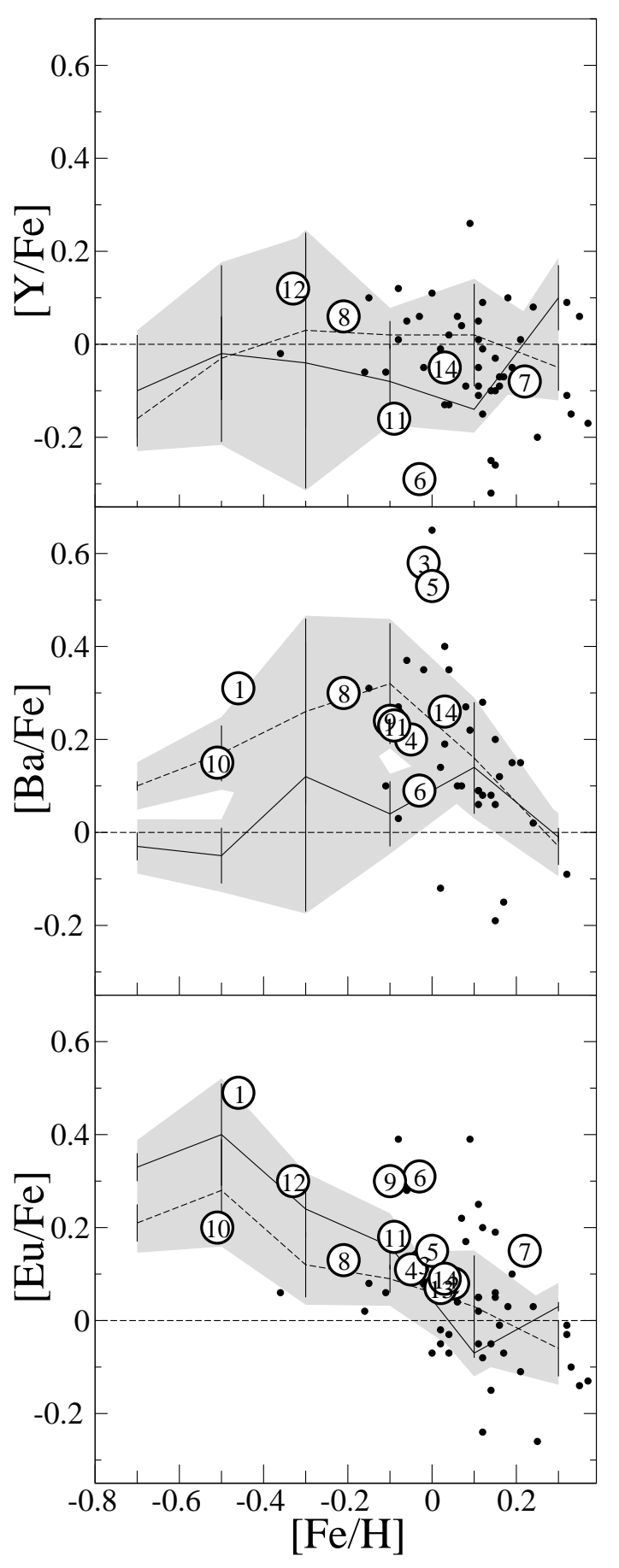

FIG. 3: [Y, Ba, Eu/Fe] abundance trends with metallicity in the atmospheres of the investigated red giants in comparison with the data for other stars. The notation is the same as that in Fig. 2.

that a substantial fraction of the program stars are located above the dwarfs that lie compactly (the shaded region). Such a behavior is also typical of the previously studied red giants and the thin-disk giants from [2] and suggests that the $[\mathrm{Na} / \mathrm{Fe}]$ abundance is determined not only by the chemical evolution of the Galaxy but also by

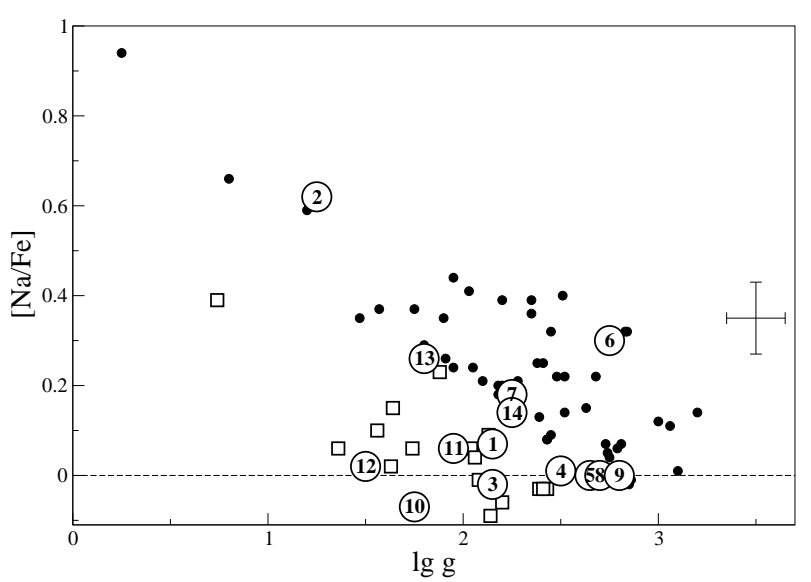

FIG. 4: $[\mathrm{Na} / \mathrm{Fe}]$ abundance versus surface gravity $\lg g$ in the atmospheres of our red giants (large open circles) and the previously investigated thin-disk (filled circles) and thick-disk (open squares) red giants. The possible error bars are indicated.

the evolution of the star itself.

The observed sodium overabundances in the atmospheres of red giants were pointed out back in 1963 [18]. When investigating the chemical composition of the red giant $\epsilon$ Vir, the authors found that the abundances of most elements were solar, within the error limits, while sodium was slightly overabundant. [30] also described the observed overabundances in the atmospheres of red giants in the Hyades open clyster. When investigating the chemical composition of red giants, the same authors 31] found several stars with an enhanced $[\mathrm{Na} / \mathrm{Fe}]$ abundance and pointed out a systematic difference in sodium abundance between red giants and dwarfs. When the results were discussed, it was hypothesized that sodium is synthesized in some red giants. In 1970, 19] ascertained that the $[\mathrm{Na} / \mathrm{Fe}]$ abundance increases with temperature parameter $\theta=5040 / T_{\text {eff }}$.

The observed sodium overabundances in the atmospheres of supergiants were detected in 1981 [11, 12]. It was then also shown that the overabundance depends on surface gravity $\lg g$ or luminosity. Subsequently, these authors hypothesized that sodium could be produced in the ${ }^{22} \mathrm{Ne}(\mathrm{p}, \gamma)^{23} \mathrm{Na}$ reaction that enters the neon-sodium cycle of hydrogen burning in the cores of main-sequence stars and could then be brought from deep layers into the stellar atmosphere through developing convection as the star evolves from the main sequence to the red giant branch [13]. The calculations performed by [21, 22] confirmed that this hypothesis is possible. Subsequent calculations using new nuclear reaction characteristics also showed a change of the sodium abundance in red giants [16, 17]. Although the neon-sodium cycle requires high temperatures and is efficient only in massive stars, low-mass $\left(1-3 M_{\odot}\right)$ stars can also produce sodium.

The stable isotope ${ }^{23} \mathrm{Na}$ is currently believed to be produced mainly in the carbon burning reaction 
${ }^{12} C\left({ }^{12} C, p\right){ }^{23} N a$. A small amount can also be produced in the hydrogen, ${ }^{22} \mathrm{Ne}(p, \gamma)^{23} \mathrm{Na}$, and helium, ${ }^{14} N(\alpha, \gamma){ }^{19} F(\alpha, \gamma){ }^{23} N a$, burning reactions [44]. The first and last reactions do not proceed in red giants at the shell hydrogen burning stage, but the second reaction is possible. Thus, the helium and carbon burning reactions in stars of earlier generations for red giants determine some basic sodium abundance, as in the case of dwarfs, and the amount of sodium produced in the stellar interior over its lifetime on the main sequence in the hydrogen burning reaction is added to this value. According to the calculations published in the review [43], the ${ }^{23} \mathrm{Na}$ abundance at a temperature $\mathrm{T}=0.05 \cdot 10^{9} \mathrm{~K}$ and a pressure of $100 \mathrm{gcm}^{-3}$ the stellar core increases by a factor of 7 in $100 \mathrm{yr}$. However, there are large uncertainties in the data on the ${ }^{22} \mathrm{Ne}(p, \gamma)^{23} \mathrm{Na}$ reaction cross sections and rates under conditions of low temperatures in the stellar cores.

Subsequently, it was shown that the observed $[\mathrm{Na} / \mathrm{Fe}]$ overabundances in the atmospheres of normal red giants also depend on surface gravity [9], with this dependence being an extension of the dependence for supergiants noted above. Interestingly, $[\mathrm{Na} / \mathrm{Fe}]$ overabundances were also detected in the atmospheres of various classes of red giants: moderate and classical barium stars [5, 14] supermetal- rich stars [39, 40], whose positions satisfy the same dependence as that for the remaining normal red giants. The subsequent sodium abundance analyses for the atmospheres of red giants performed at the Institute of Astronomy, the Russian Academy of Sciences, showed that some stars have a reduced $[\mathrm{Na} / \mathrm{Fe}]$ abundance relative to the observed dependence on surface gravity [6, 39, 40]. The kinematic characteristics of such stars correspond to thick-disk objects.

In Fig. 2, HD 10550 lies above the remaining stars and has a sodium abundance that is higher than that for dwarfs at the same metallicity approximately by 0.5 dex. Among all of our program stars, HD 10550 has the lowest surface gravity $\lg g=1.25$. HD 152879 also lies statistically above the dwarfs $(\lg g=1.80)$. The remaining stars lie, within the error limits, in the region where the dwarfs are located, except HD 127243; it has a [Na/Fe] abundance that is lower than that for dwarfs approximately by 0.15 dex.

In Fig. 4 , the $[\mathrm{Na} / \mathrm{Fe}]$ abundance is plotted against the surface gravity $\lg g$ in the atmospheres of the program stars in comparison with the previously studied thin-and thick-disk red giants. We see that the positions of the thin-disk stars form a distinct dependence, while the thick-disk stars with a reduced $[\mathrm{Na} / \mathrm{Fe}]$ abundance lie below it. In this figure, most of the program stars are also located below the dependence for the thin-disk giants in the region where the thick-disk stars dominate. For some of the stars with a relatively low metallicity (HD $127243[\mathrm{Fe} / \mathrm{H}]=-0.44$ and $\mathrm{HD} 141472[\mathrm{Fe} / \mathrm{H}]=-0.27)$, the $[\mathrm{Na} / \mathrm{Fe}]$ underabundance reaches 0.5 dex. The stars from Table \ with a high probability of their membership in the thin disk but with a large orbital parameter
$Z_{\max }$ (HD 94600, HD 99902, and HD 100696) are located at the lower boundary of the region for the thin-disk giants and at its upper boundary for the thick-disk giants. In this case, we cannot determine with confidence which dynamical subsystem of the Galaxy they belong to from Fig. 4.

The causes of the reduced sodium abundance in the atmospheres of thick-disk red giants relative to the $[\mathrm{Na} / \mathrm{Fe}]-\lg g$ relation can be: (1) the initial sodium underabundance; (2) the initial neon under- abundance that led to the production of a smaller amount of sodium; and (3) the shallower convective shell. The first assumption is not backed by the observational data; the $[\mathrm{Na} / \mathrm{Fe}]$ abundances in the thick- and thin-disk dwarfs differ only slightly. The second assumption can possibly play a significant role, but it is difficult to test this hypothesis at present due to the absence of accurate data on the neon abundances in thick-disk stars. The last assumption is unlikely, because the physical parameters of the stars $\left(T_{\text {eff }}, \lg g,[\mathrm{Fe} / \mathrm{H}]\right.$, mass) with normal and reduced $[\mathrm{Na} / \mathrm{Fe}]$ abundances are identical and it would be unreasonable to expect the convective processes in them to differ noticeably.

Among the program thick-disk stars, there are two with a significant $[\mathrm{Na} / \mathrm{Fe}]$ abundance relative to other stars with similar parameters. The first star HD 94860 is located in a region characteristic of the metal-rick thin-disk stars. For example, the Hyades giants (with $[\mathrm{Fe} / \mathrm{H}] \approx 0.14 \mathrm{dex})$ are also located there. The equivalent widths of the sodium 6154 and $6160 \AA$ lines are 85 and $105 \mathrm{~m} \AA$, respectively; such very close values were also measured for HD 94669 with similar $\lg g$ and $[\mathrm{Fe} / \mathrm{H}]$, but this star has a much lower temperature (by $250 \mathrm{~K}$ ) and, hence, a low abundance $[\mathrm{Na} / \mathrm{Fe}]=0.00$ dex. Since the errors in the $[\mathrm{Na} / \mathrm{Fe}]$ abundance (see Table IV) cannot change it greatly, the estimated sodium abundance in HD 94860 is realistic. This star, which kinematically belongs to the thick disk, may have been formed in agas.dust cloud similar in chemical composition to the thin-disk objects. The second star HD 10500 is located in Fig. 4 near the thin-disk supergiants. However, it has the highest mass among the program stars $\left(7 M_{\odot}\right)$ and, consequently, the internal physical conditions under which sodium is synthesized more efficiently. The star may also have a deeper convective envelope that provides the rise of sodium from the stellar interior.

\section{Magnesium}

In Fig. 2, all of the program stars are located, within the magnesium abundance error limits, in the region of dwarfs. Three red giants with the lowest iron abundance (HD 127243, HD 2901, and HD 141472) clearly correspond to the distribution of thick-disk stars. In this region with $[\mathrm{Fe} / \mathrm{H}] \lesssim-0.3$, the separation of the thin-and thick-disk dwarfs is largest (about 0.2 dex) and exceeds the mean abundance errors (about $0.1 \mathrm{dex}$ ). The 
data on the giants from [2] also closely correspond to the trends for the dwarfs: the thin-disk giants lie along the solid line, while the thick-disk giants lie along the dashed line.

Magnesium is produced in the carbon, ${ }^{16} \mathrm{O}\left({ }^{12} \mathrm{C}, \alpha\right){ }^{24} \mathrm{Mg}, \quad$ helium, $\quad{ }^{20} \mathrm{Ne}(\alpha, \gamma)^{24} \mathrm{Mg}$, ${ }^{21} \mathrm{Ne}(\alpha, n)^{24} \mathrm{Mg}$, and hydrogen, ${ }^{23} \mathrm{Na}(p, \gamma){ }^{24} \mathrm{Mg}$, ${ }^{27} \mathrm{Al}(p, \alpha){ }^{24} \mathrm{Mg}$, burning reactions. The case of carbon and helium burning plays no role in the chemical evolution during the lifetime of red giants, while the last hydrogen burning reaction in the magnesium. aluminum cycle is mots likely inefficient, because this requires conditions with much higher temperatures than those in low-mass stars at the red giant stage. Only the reaction with sodium involvement ${ }^{23} \mathrm{Na}(p, \gamma)^{24} \mathrm{Mg}$ can slightly raise the magnesium abundance [43]. This may underlie the slight $[\mathrm{Mg} / \mathrm{Fe}]$ overabundance for some normal red giants. However, the entire effect is within the $2 \sigma$ magnesium abundance error limits.

\section{Aluminum}

In Fig. 2, all of the program stars are located, within the aluminum abundance error limits, in the region of dwarfs. Just as in the case of magnesium, three red giants with the lowest iron abundance (HD 127243, HD 2901, and HD 141472) clearly correspond to the distribution of thick-disk dwarfs. The data from [2] show a larger scatter of abundances relative to the dwarfs than that for magnesium. Some of the previously studied red giants are located approximately 0.2 dex higher than the dwarfs. Although this difference does not exceed the $2 \sigma$ level, the published data suggest an enhanced aluminum abundance in globular cluster giants [16].

In stars, aluminum can be produced in two reactions of the magnesium.aluminum cycle of hydrogen burning: ${ }^{26} \mathrm{Mg}(p, \gamma){ }^{27} \mathrm{Al}$ and ${ }^{26} \mathrm{Al}(p, \gamma){ }^{27} \mathrm{Si}\left(\beta^{+} \nu_{e}\right)^{27} \mathrm{Al}$. The physical parameters needed for the ${ }^{27} A l$ production reaction were estimated by [26], who described the possibility of the existence of observable aluminum overabundances in the atmospheres of red giants.

\section{Silicon}

In Fig. 2, all of the program stars are located within the silicon abundance error limits, in the region of dwarfs. Just as in the case of magnesium and aluminum, three red giants with the lowest iron abundance (HD 127243, HD 2901, and HD 141472) correspond to the distribution of thick-disk dwarfs.

The isotope ${ }^{28} \mathrm{Si}$ is produced mainly in the oxygen burning reaction ${ }^{16} O\left({ }^{16} \mathrm{O}, \alpha\right){ }^{28} \mathrm{Si}$, which is inaccessible for low-mass red giants. The ${ }^{27} \mathrm{Al}(p, \gamma)^{28} \mathrm{Si}$ reaction entering the magnesium.aluminum cycle of hydrogen burning can be a possible silicon production source, but its contribution to nucleosynthesis in low-mass stars is negligible and is considered as a leakage reaction [16].

\section{Calcium}

In Fig. 2, almost all of the program stars are located, within the calcium abundance error limits, in the region of dwarfs. Just as in the case of magnesium and aluminum, three red giants with the lowest iron abundance (HD 127243, HD 2901, and HD 141472) correspond to the distribution of thick-disk dwarfs.

Calcium is an $\alpha$-element and it originates in the oxygen burning reactions. The calcium abundance does not change over the lifetime of a red giant.

\section{Titanium}

Titanium, along with magnesium, silicon, and calcium, is an $\alpha$-element and, at the same time, also belongs to the iron-peak elements produced during supernova 1a explosions. Just as other .elements, titan is characterized by an increase in its abundance with decreasing metallicity. Just as in the case of magnesium, aluminum, silicon, and calcium, three red giants with the lowest iron abundance (HD 127243, HD 2901, and HD 141472) correspond to the distribution of thick-disk dwarfs. A slightly overestimated titanium abundance was determined for HD 94860 , but it is within the $1 \sigma$ error limits relative to the dwarfs.

\section{Yttrium}

As one of the s-elements, yttrium is produced in the slow neutron capture process. The presence of a flux of free neutrons is necessary for this process. This condition is met in stars at the asymptotic giant branch stage.a later evolutionary stage relative to the normal red giants. Therefore, the same picture as that for the dwarf stars must be observed (Fig. 3).

However, this figure does not present the moderate and classical barium stars investigated previously [5, 14]. In classical barium stars, binarity plays a major role. one of the binary components evolves more rapidly and ejects its envelope rich in s-elements part of which is captured by the secondary component. They are characterized by a different, steeper trend with decreasing metallicity [5]. In contrast, for moderate barium stars, some of which can be represented by single red giants, possible photoneutron reactions like ${ }^{13} C(\gamma, n){ }^{12} C$ and ${ }^{14} N(\gamma, n){ }^{13} N$ can play a role. According to 29], when the CNO cycle of hydrogen burning proceeds in the interior of a mainsequence star, a large number of energetic gamma- ray photons capable of knocking out a neutron from the nuclei of some elements emerge. This source of neutrons is weak, but, as was pointed out by [29], it is the second 
in intensity among all of the known neutron sources and could possibly explain the small anomalies of s-process elements observed in stars at the red giant stage.

\section{Barium}

In Fig. 3, all of the program stars, except two, lie in the region of dwarfs. These stars (HD 92095 and HD 94669) have the most saturated barium lines among the stars presented on the plot and probably the largest errors in the barium abundance. The most metal-poor stars HD 127243 and HD 2901 are located in a region characteristic of the thick-disk dwarfs.

\section{Europium}

Europium is an r-element whose origin is associated with the rapid neutron capture process that occurs during supernova explosions, and its abundance does not change during the evolution of a red giant. Within the error limits, the program stars are located in the same region as the dwarfs and exhibit an increase in $[\mathrm{Eu} / \mathrm{Fe}]$ abundance with decreasing metallicity.

\section{CONCLUSIONS}

We investigated 14 red giants with high space velocities. For all stars, we determined their Galactic orbital elements and other kinematic characteristics from which their membership in a particular Galactic subsystem can be judged. We determined the physical parameters, stellar atmosphere parameters, and elemental abundances. For almost all chemical elements, their abundance trends with metallicity were shown to correspond to those for dwarfs. Where a significant difference exists between the trends corresponding to the thin-and thickdisk dwarfs, the positions of the thick-disk giants follow the trend for the thick-disk dwarfs. An abundance analysis of the atmospheres of red giants provides an additional possibility to determine whether a star belongs to the Galactic thick or thin disk in some cases. A different picture is observed for sodium: in addition to the dependence on metallicity, which is determined by the chemical evolution of the Galaxy, there is a significant dependence on surface gravity $\lg g$, which reflects the degree of development of the convective envelope in a red giant. In this case, there is a significant difference between the $[\mathrm{Na} / \mathrm{Fe}]$ abundances in the atmospheres of thick-and thin-disk red giants.

Acknowledgments This work was supported by the Russian Foundation for Basic Research (projects no. 0902-00528-a, 12-02-00610-a), the "Origin, Structure, and Evolution of Objects in the Universe". Program of the Presidium of the Russian Academy of Sciences, and the Federal Agency for Science and Innovations (State contract no. 02.740.11.0247).
[1] Allen, C. and Santillan, A., Revista Mexicana de Astronomia y Astrofisica 22, 255 (1991).

[2] Alves-Brito, A., Meléndez, J., Asplund, M., Ramírez, I., and Yong, D., Astron. Astrophys. 513, A35+ (2010) arXiv:1001.2521 [astro-ph.SR]

[3] Antipova, L. I. and Boyarchuk, A. A., Astronomy Reports 45, 700 (2001)

[4] Antipova, L. I., Boyarchuk, A. A., Pakhomov, Y. V., and Panchuk, V. E., Astronomy Reports 47, 648 (2003)

[5] Antipova, L. I., Boyarchuk, A. A., Pakhomov, Y. V., and Panchuk, V. E., Astronomy Reports 48, 597 (2004)

[6] Antipova, L. I., Boyarchuk, A. A., Pakhomov, Y. V., and Yushkin, M. V., Astronomy Reports 49, 535 (2005)

[7] Bensby, T., Feltzing, S., Lundström, I., and Ilyin, I., Astron. Astrophys. 433, 185 (2005).

[8] Bessell, M. S., Castelli, F., and Plez, B., Astron. Astrophys. 333, 231 (1998).

[9] Boyarchuk, A. A., Antipova, L. I., Boyarchuk, M. E., and Savanov, I. S., Astronomy Reports 45, 301 (2001)

[10] Boyarchuk, A. A., Antipova, L. I., and Pakhomov, Y. V., Astronomy Reports 52, 630 (2008)

[11] Boyarchuk, A. A. and Boyarchuk, M. E., Bulletin Crimean Astrophysical Observatory 63, 68 (1981).
[12] Boyarchuk, A. A. and Lyubimkov, L. S., Bulletin Crimean Astrophysical Observatory 64, 1 (1981).

[13] Boyarchuk, A. A. and Lyubimkov, L. S., Bulletin Crimean Astrophysical Observatory 66, 119 (1983).

[14] Boyarchuk, A. A., Pakhomov, Y. V., Antipova, L. I., and Boyarchuk, M. E., Astronomy Reports 46, 819 (2002).

[15] Castelli, F. and Kurucz, R. L., in Modelling of Stellar Atmospheres, IAU Symposium, Vol. 210, edited by N. Piskunov, W. W. Weiss, and D. F. Gray (2003) pp. 20P-+.

[16] Cavallo, R. M., Sweigart, A. V., and Bell, R. A., Astrophys. J. 464, L79+ (1996).

[17] Cavallo, R. M., Sweigart, A. V., and Bell, R. A., Astrophys. J. 492, 575 (1998).

[18] Cayrel, G. and Cayrel, R., Astrophys. J. 137, 431 (1963)

[19] Cayrel de Strobel, G., Chauve-Godard, J., Hernandez, G., and Vaziaga, M. J., Astron. Astrophys. 7, 408 (1970).

[20] Chen, B., Stoughton, C., Smith, J. A., Uomoto, A., Pier, J. R., Yanny, B., Ivezić, Ž., York, D. G., Anderson, J. E., Annis, J., Brinkmann, J., Csabai, I., Fukugita, M., Hindsley, R., Lupton, R., Munn, J. A., and the SDSS Collaboration,, Astrophys. J. 553, 184 (2001). 
[21] Denisenkov, P. A., Soviet Astronomy Letters 14, 435 (1988).

[22] Denisenkov, P. A. and Ivanov, V. V., Soviet Astronomy Letters 13, 214 (1987).

[23] Eggen, O. J., Monthly Notices Roy. Astron. Soc. 118, 65 (1958).

[24] Eggen, O. J., Monthly Notices Roy. Astron. Soc. 118, 154 (1958).

[25] Famaey, B., Jorissen, A., Luri, X., Mayor, M., Udry, S., Dejonghe, H., and Turon, C., Astron. Astrophys. 430, 165 (2005)

[26] Fujimoto, M. Y., Aikawa, M., and Kato, K., Astrophys. J. 519, 733 (1999).

[27] Gilmore, G. and Reid, N., Monthly Notices Roy. Astron. Soc. 202, 1025 (1983).

[28] Girardi, L., Bressan, A., Bertelli, G., and Chiosi, C., Astron. Astrophys. Suppl. Ser. 141, 371 (2000).

[29] Harrison, T. G. and Edwards, T. W., Astrophys. J. 187, 303 (1974).

[30] Helfer, H. L. and Wallerstein, G., Astrophys. J. Suppl. Ser. 9, 81 (1964).

[31] Helfer, H. L. and Wallerstein, G., Astrophys. J. Suppl. 16, 1 (1968).

[32] Korotin, S. A. and Komarov, N. S., Soviet Astronomy 33, 449 (1989).

[33] Kurucz, R., ATLAS9 Stellar Atmosphere Programs and 2 km/s grid. Kurucz CD-ROM No. 13. Cambridge, Mass.: Smithsonian Astrophysical Observatory, 1993. 13 (1993).
[34] Lind, K., Asplund, M., Barklem, P. S., and Belyaev, A. K., Astron. Astrophys. 528, A103+ (2011), arXiv:1102.2160 [astro-ph.SR].

[35] Mashonkina, L. I., Shimanskii, V. V., and Sakhibullin, N. A., Astronomy Reports 44, 790 (2000).

[36] Maurice, E., Andersen, J., Ardeberg, A., Bardin, C., Imbert, M., Lindgren, H., Martin, M., Mayor, M., Nordstrom, B., Prevot, L., Rebeirot, E., and Rousseau, J., Astron. Astrophys. Suppl. Ser. 67, 423 (1987).

[37] Mishenina, T. V., Soubiran, C., Kovtyukh, V. V., and Korotin, S. A., Astron. Astrophys. 418, 551 (2004).

[38] Pakhomov, Y. V., Antipova, L. I., and Boyarchuk, A. A., Astronomy Reports 55, 256 (2011).

[39] Pakhomov, Y. V., Antipova, L. I., Boyarchuk, A. A., Bizyaev, D. V., Zhao, G., and Liang, Y., Astronomy Reports 53, 660 (2009).

[40] Pakhomov, Y. V., Antipova, L. I., Boyarchuk, A. A., Zhao, G., and Liang, Y., Astronomy Reports 53, 685 (2009).

[41] Robin, A. C., Haywood, M., Creze, M., Ojha, D. K., and Bienayme, O., Astron. Astrophys. 305, 125 (1996).

[42] van Leeuwen, F., Astron. Astrophys. 474, 653 (2007).

[43] Wallerstein, G., Iben, I., Parker, P., Boesgaard, A. M., Hale, G. M., Champagne, A. E., Barnes, C. A., Käppeler, F., Smith, V. V., Hoffman, R. D., Timmes, F. X., Sneden, C., Boyd, R. N., Meyer, B. S., and Lambert, D. L., Rev. Mod. Phys. 69, 995 (1997).

[44] Woosley, S. E. and Weaver, T. A., Astrophys. J. Suppl. Ser. 101, 181 (1995). 